\title{
An analysis of recent stroke cases in South Africa: Trend, seasonality and predictors
}

\author{
E Ranganai, ${ }^{1} \mathrm{BSc}, \mathrm{BSc}$ Hons, MSc, PhD; L Matizirofa, ${ }^{2} \mathrm{BSc}, \mathrm{MSc}$ \\ ${ }^{1}$ Department of Statistics, College of Science, Engineering and Technology, School of Science, University of South Africa, Roodepoort, South Africa \\ ${ }^{2}$ Department of Statistics, Faculty of Science, University of Johannesburg, South Africa
}

Corresponding author: E Ranganai (rangae@unisa.ac.za)

\begin{abstract}
Background. South Africa (SA) is experiencing an epidemiological transition as a result of sociodemographic and lifestyle changes. This process is leading to an increase in non-communicable diseases, which in turn may result in an upswing of stroke cases. Stroke is among the top 10 leading causes of disability in SA, and accounts for 25000 deaths annually and 95000 years lived with disability (YLD). This huge burden of stroke hampers socioeconomic development as a result of YLD.

Objectives. To investigate the seasonality and trend of stroke cases in SA, and determine the risk factors associated with stroke.

Methods. Using recent hospital-based data (January 2014 - December 2017 inclusive) from SA private and public hospitals (33\% private and $67 \%$ public), a sample of 14645 suspected stroke cases was drawn. Associations between suspected stroke cases and potential predictors were assessed using $\chi^{2}$ tests and bivariate analysis. Time series analysis tools for trend and seasonality components included both time domain and frequency domain techniques. A Poisson generalised linear model was used, as there was no over-dispersion inherent in the data. Multiple logistic regression analysis was used to assess the effect of several predictors on stroke cases.

Results. Of the 14645 suspected cases of stroke, 51.5\% were confirmed. Seasonality analysis gave an approximate seasonal change of 120 cases, the highest seasonal peak occurring in mid-winter and the lowest dip in mid-summer. Both upward trend and seasonality parameters were found to be statistically significant. Predictors significantly associated with an increased likelihood of stroke were heart problems (odds ratio (OR) 8.86; 95\% confidence interval (CI) 8.23 - 9.55; $p<0.0001$ ), diabetes (OR 14.53; 95\% CI 13.36 - 15.79; $p<0.0001$ ), female sex (OR 18.23; 95\% CI 16.75 - 19.85; $p<0.0001$ ), age 59 - 77 years (OR 1.37; 95\% CI 1.24 - 1.50; $p<0.0001$ ) and 78 - 98 years (OR 1.25; $95 \%$ CI $1.16-1.35 ; p<0.0001$ ) and white ethnic group (OR 2.00; 95\% CI $1.86-2.15$; $p<0.0001$ ), compared with the respective reference groups. The prevalence ratios of stroke cases as measured by Poisson regression were in agreement with logistic regression results.

Conclusions. The increasing trend of stroke in SA should be arrested urgently, taking into account both the associated risk factors and seasonality.
\end{abstract}

S Afr Med J 2020;110(2):92-99. https://doi.org/10.7196/SAMJ.2020.v110i2.13891

Globally, stroke is the second leading cause of mortality and the third leading cause of disability. ${ }^{[1,2]}$ African countries are experiencing an epidemiological transition as a result of sociodemographic and lifestyle changes. ${ }^{[3]}$ This process is leading to an increase in non-communicable diseases. In the case of South Africa (SA), this transition, which significantly increased at the advent of democracy in 1994, continues unabated. In sub-Saharan Africa, which includes SA, the burden of stroke is on the rise ${ }^{[1,2,4]}$ owing to increasing cases. Stroke is among the top 10 leading causes of disability in SA, ${ }^{[5,6]}$ accounting for 25000 deaths a year and 95000 years lived with disability (YLD). ${ }^{[7]}$ This huge burden of stroke hampers socioeconomic development as a result of YLD. Furthermore, Mudzi et al. ${ }^{[5]}$ found that $25.5 \%$ of stroke patients died within 3 months of discharge and $38 \%$ within 12 months. As in other low- to middle-income countries (LMICs), including those in Africa, development of effective advocacy strategies for policy action in SA is curtailed by insufficient and/or inaccurate information due to scanty data available.

Risk factors for stroke can be classified as modifiable or nonmodifiable. ${ }^{[4]}$ Modifiable factors are the controllable ones such as hypertension, raised cholesterol, smoking, obesity, physical inactivity, heart disease and alcohol abuse, while non-modifiable (natural) risk factors include age, gender, genetics, ethnic group and family history of stroke. A study by Ovbiagele and Nguyen-Huynh ${ }^{[8]}$ identified elevated blood pressure as a dominant risk factor in African Americans, with other modifiable lifestyle risk factors including heavy alcohol consumption, raised cholesterol, physical inactivity and smoking. Raised cholesterol was found to be the dominant risk factor in white females. In a recent study by Wandai et al. ${ }^{[9]}$ tobacco use, hypertension, obesity, raised cholesterol, physical inactivity and unhealthy diets were identified as the major risk factors for stroke in SA. The lifestyle changes associated with urbanisation include bad dietary habits and high stress levels, resulting in a host of noncommunicable diseases that in turn contribute to increasing cases of stroke. Efforts to improve the situation are hampered by the fact that the urban healthcare infrastructure was not designed to cater for this rapidly growing population. Furthermore, government programmes to encourage awareness of risk factors for stroke are lacking. For the government to make informed decisions about such programmes, comprehensive studies on stroke still need to be undertaken.

In Europe, North America and Australia, where extensive quality data are available, many studies on non-communicable diseases have been carried out in order to improve understanding of the problem. In LMICs, however, including those in Africa, data remain scanty, adversely affecting the quality and extent of such studies. Studies on seasonality and trends of stroke and non-communicable diseases, and associated risk factors, are mostly based on Western populations. 
A season is defined as a pattern in a health outcome or exposure that increases or decreases with some regularity. ${ }^{[10]}$ Seasonality and climatic features in stroke cases have been extensively studied in Iran. ${ }^{[4]}$ The seasonality of stroke cases and mortality has been extensively evaluated in many Western but few African countries. ${ }^{[11]}$ Most studies have reported increased cases of stroke in winter, ${ }^{[1-14]}$ fewer noting a high proportion of cases in summer. ${ }^{[15]}$

Precise information on stroke cases, seasonality, trends and risk factors from hospital-based data is needed to develop public health interventions such as awareness campaigns on risk factors and possible measures to reduce the burden of stroke. In SA, such studies are urgently needed to obtain this critical information.

\section{Objectives}

To the best of the authors' knowledge, there has been no published research on seasonal variations in cases of stroke in SA to date. The primary objectives of this study were to identify the seasonality and trend of stroke in SA and determine the associated risk factors for stroke, using data retrieved from randomly selected private and public hospitals between January 2014 and December 2017 inclusive.

\section{Methods \\ Study design}

A cross-sectional study design was used to explore the seasonality and trend of stroke cases in SA for January 2014 - December 2017. This was a descriptive epidemiological study in which the exposure and disease status of the population were determined at a given point. The study design chosen was aimed at obtaining immediate knowledge and information about the seasonal pattern of stroke cases. The study covered the years 2014 - 2017 because SA hospitals keep patients' folders for only 3 years (plus the records for 2017 of the next 3-year cycle) in hospital storage rooms before sending them to outside storage.

\section{Data collection}

Data on suspected stroke patients aged $\geq 18$ years were retrieved from randomly selected public and private hospitals (33\% private and $67 \%$ public) in the nine provinces of SA, namely Eastern Cape, Free State, Gauteng, KwaZulu-Natal, Limpopo, Mpumalanga, Northern Cape, North West and Western Cape, between January 2014 and December 2017. Although most South Africans ( 70\%) use public hospitals, many of these institutions did not capture all the pertinent variables, while the private hospitals were doing so. The case managers for the sampled hospitals assisted with data retrieval. The total number of suspected strokes, including non-confirmed strokes, was 14645 . Stroke cases $(N=7538)$ were confirmed by computed tomography or magnetic resonance imaging.

\section{Study variables}

\section{Dependent variable}

The response variable was confirmed stroke, and it was coded $1=$ yes and $0=$ no.

\section{Independent variables}

The explanatory variables are shown in Table 1. This table shows that the risk factors and demographic variables assessed are significantly associated with stroke.

\section{Study setting}

This study was carried out in SA (population $~ 57$ million). SA comprises nine provinces. Table 2 shows the population estimates by province.
There are 407 public hospitals (67\%) and 203 private hospitals (33\%) in SA. Stratified random sampling was used to calculate the proportions accordingly, i.e. $33 \%$ of the 203 private hospitals and $67 \%$ of the 407 public hospitals were sampled for the study.

\section{Ethical considerations}

Permission to conduct this research was obtained from the provincial departments, from the individual hospitals and from the Committee for Research on Human Subjects of the University of South Africa (ref. no. 2017/SSR-ERC/001).

\section{Statistical analysis}

Various statistical techniques have been used to examine seasonal variations in disease occurrence, but we used a Poisson generalised linear model (GLM) for count time series data, since stroke cases are count data. GLMs are statistical models that provide a flexible approach in seasonality studies, allowing data to be fitted to numerous mathematical functions. ${ }^{[10]}$ Simple logistic and Poisson regressions were used to explore the relationship of individual predictor variables with stroke cases. Finally, multivariate logistic regression analysis was used to identify the effect of several independent predictors on stroke cases.

Quantitative data analysis was done using $\mathrm{R}$ statistical software, version 3.4.3 (R Foundation for Statistical Computing). Descriptive statistics were computed to describe data characteristics of stroke cases. The $\mathrm{R}$ add-on packages 'season' and 'ts count' were used for fitting the Poisson GLM from which seasonality and trend analysis and Poisson regression were carried out. Both monthly time series and circular mean plots of monthly cases of stroke adjusted to a 30-day month were done. Simple logistic regression analysis was used to show how each predictor contributed to the total variability of stroke disease. Multivariate logistic regression analysis was used to identify the effect of several independent predictors of stroke.

\section{Definition of season for the study}

The four seasons were defined according to the SA meteorological reports as winter (June, July, August), spring (September, October, November), summer (December, January, February) and autumn (March, April, May). This study used these four defined seasons.

\section{Results}

Demographic characteristics of the suspected stroke patients are shown in Table 1. A total of 14645 suspected stroke patients were retrieved from the private and public hospitals for the period 2014 2017, of whom $57.2 \%$ were female and $42.8 \%$ were male. The majority were married (67.5\%), and very few were divorced (3.9\%). A large proportion (69.6\%) were white, $27.3 \%$ were Asians, $3.1 \%$ were black, and there were no Indians or coloureds. To address this data sparsity problem all the other races were compared against the white race. A large number of suspected stroke patients lived in urban areas (99.8\%). The mean (standard deviation) age for the suspected stroke patients was 66.95 (19.48) years (Table 3). Table 3 shows a range of age of 18 98 years $(98-18=80)$, which is an indication of high variation (with a total of 14645$)$.

The largest number of confirmed stroke cases was recorded in 2017 ( $n=2$ 328) (Table 4). Numbers of cases show an upward trend between the years 2014 and 2017, at 1491, 1 778, 1941 and 2 328, respectively.

Table 5 shows that diabetes and heart problems were risk factors in larger numbers of cases of stroke than hypertension and raised cholesterol. These findings were similar to those of a recent study in Iran. ${ }^{[1]}$ While we found the prevalence of diabetes to be high in 
Table 1. The $\chi^{2}$ test of association between stroke and potential predictors

\begin{tabular}{|c|c|c|c|}
\hline & Suspected stroke $(N=14645), n(\%)$ & Confirmed stroke ( $N=7538), n(\%)$ & $p$-value \\
\hline \multicolumn{4}{|l|}{ Demographic variables } \\
\hline Gender & & & $<0.0001$ \\
\hline Male & $6270(42.8)$ & $1015(13.5)$ & \\
\hline Female & $8375(57.2)$ & $6523(86.5)$ & \\
\hline Race & & & $<0.0001$ \\
\hline Black & $455(3.1)$ & $453(6.0)$ & \\
\hline White & $10182(69.5)$ & $4312(57.2)$ & \\
\hline Indian & 0 & 0 & \\
\hline Coloured & 0 & 0 & \\
\hline Asian & $4008(27.4)$ & $2773(36.8)$ & \\
\hline Marital status & & & $<0.0001$ \\
\hline Single & $2162(14.8)$ & $2163(28.7)$ & \\
\hline Married & $9876(67.4)$ & $3951(52.4)$ & \\
\hline Divorced & $576(3.9)$ & $111(1.5)$ & \\
\hline Widow & $1595(10.9)$ & $877(11.6)$ & \\
\hline Other & $436(3.0)$ & $436(5.8)$ & \\
\hline Place of residence & & & $<0.0001$ \\
\hline Rural & $22(0.2)$ & 0 & \\
\hline Urban & $14622(99.8)$ & $7538(100)$ & \\
\hline Informal settlement & 0 & 0 & \\
\hline Other & $1(0.007)$ & 0 & \\
\hline \multicolumn{4}{|l|}{ Risk factors for stroke } \\
\hline Hypertension & & & $<0.0001$ \\
\hline Yes & $5401(36.9)$ & $3755(49.8)$ & \\
\hline No & $9244(63.1)$ & $3783(50.2)$ & \\
\hline Raised cholesterol & & & $<0.0001$ \\
\hline Yes & $6248(42.7)$ & $3610(47.9)$ & \\
\hline No & $8397(57.3)$ & $3928(52.1)$ & \\
\hline Heart problems & & & $<0.0001$ \\
\hline Yes & $7107(48.5)$ & $5710(75.7)$ & \\
\hline No & $7538(51.5)$ & $1828(24.3)$ & \\
\hline Diabetes & & & $<0.0001$ \\
\hline Yes & 7107 (48.5) & $6550(86.9)$ & \\
\hline No & $7538(51.5)$ & $988(13.1)$ & \\
\hline
\end{tabular}

Table 2. Mid-year population estimates by province, $2015^{[16]}$

\begin{tabular}{lll}
\hline Province & $\begin{array}{l}\text { Population } \\
\text { estimate, } \boldsymbol{n}\end{array}$ & \% of total population \\
\hline Eastern Cape & 6916200 & 12.6 \\
Free State & 2817900 & 5.1 \\
Gauteng & 13200300 & 24.0 \\
KwaZulu-Natal & 10919100 & 19.9 \\
Limpopo & 5726800 & 10.4 \\
Mpumalanga & 4283900 & 7.8 \\
Northern Cape & 1185600 & 2.2 \\
North West & 3707000 & 6.7 \\
Western Cape & 6200900 & 11.3 \\
Total & 54956900 & 100 \\
& &
\end{tabular}

white female stroke patients, hypertension was found to be the most common risk factor in black females. Table 5 indicates considerable monthly variation in risk factors, with high proportions of cases in patients with hypertension, diabetes and heart problems in the winter months, in accordance with the findings of Bahonar et al. ${ }^{[1]}$ and Wang
Table 3. Summary statistics of a continuous variable, age (years)

\begin{tabular}{ll}
\hline Statistic & Statistic value \\
\hline Mean & 66.95 \\
SD & 19.48 \\
Minimum & 18 \\
Maximum & 98 \\
Range & 80 \\
SD = standard deviation. &
\end{tabular}

Table 4. Numbers of confirmed strokes over the 4-year study period

\begin{tabular}{ll}
\hline Year & Confirmed strokes, $\boldsymbol{n}$ \\
\hline 2014 & 1491 \\
2015 & 1778 \\
2016 & 1941 \\
2017 & 2328
\end{tabular}

et al. ${ }^{[11]}$ In contrast, there were few stroke patients with these risk factors in the month of November. 


\section{Analysis of monthly seasonal patterns}

Monthly numbers of confirmed stroke cases in adults aged $\geq 18$ years in SA for the years $2014-2017$ are shown in Fig. 1. A general upward trend is evident, as well as regular peaks suggesting inherent seasonality. The results of a formal test for seasonality are shown in Table 6, which shows the size of the seasonal change (amplitude) and the seasonality of the highest peak and the lowest dip. This

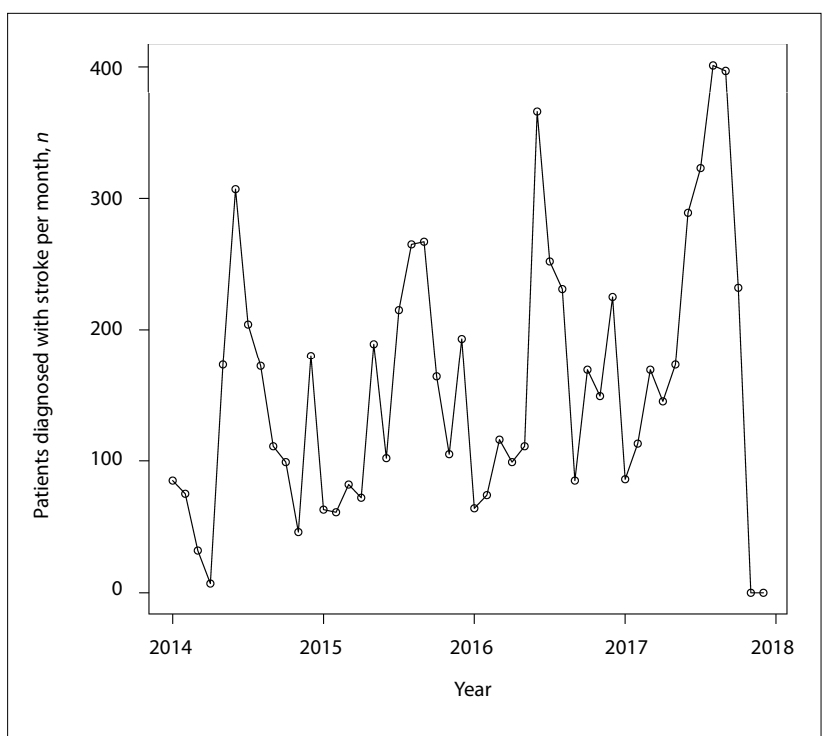

Fig. 1. Time series plot of confirmed stroke cases in South Africa, 2014 - 2017.

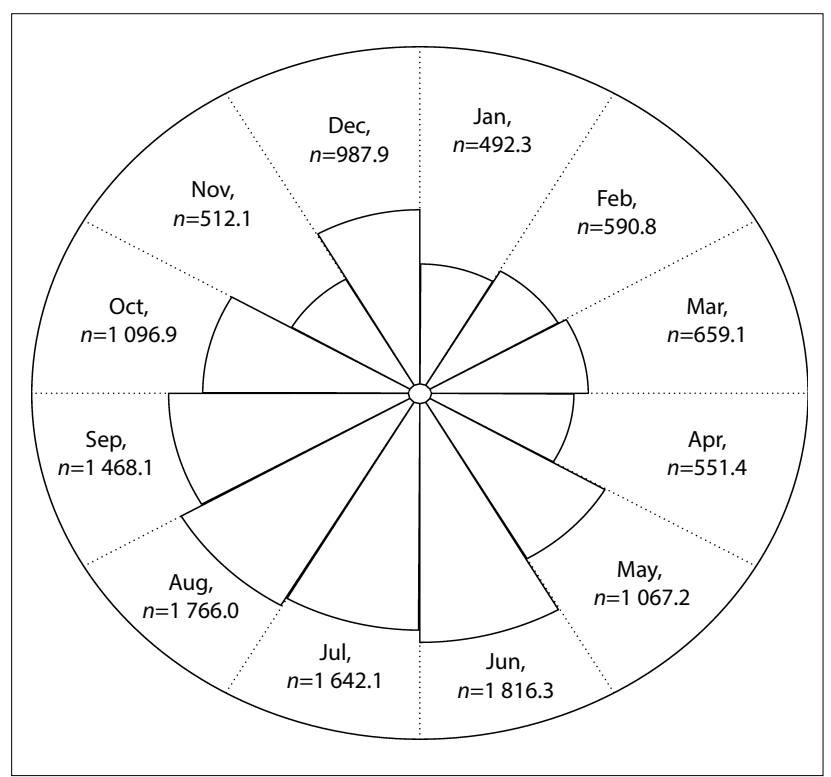

Fig. 2. Circular plot of monthly suspected stroke cases with data adjusted to a 30-day month, 2014 - 2017. corresponds to an approximate seasonal change of 120 cases, the highest seasonal peak occurring in mid-winter (approximately midway between July and August) and the lowest dip in mid-summer (approximately midway between December and January).

A circular plot of mean monthly cases of stroke adjusted to a common month length of 30 days $^{[10]}$ is shown in Fig. 2. The highest and lowest monthly mean rates correspond to June and January, respectively. These results are consistent with those of other studies $^{[14,11]}$ showing seasonal variation in stroke cases, with a higher rate in winter and a lower rate in summer. In the literature, most studies indicate stroke cases to be at their peak in winter, followed by spring.

To investigate whether the mean ratios of all the other months to the lowest reference month of January were statistically different from zero, a Poisson GLM was fitted. The results are shown in Table 7. All the mean ratios are statistically different from 1 as shown by their $95 \%$ confidence intervals (CIs), which exclude 1 $(p<0.05)$, except for the two months of April and November. Note that the highest mean rate of stroke cases in June is $\sim 3.69$ times the lowest one in January.

The results shown in Table 7 are displayed graphically in Fig. 3, which clearly shows that all the mean ratios do not cross 1 , the January level, except for the two months of April and November, confirming the interpretation given for the results in Table 7.

\section{Non-stationary seasonality model}

Finally, to check for changing (non-stationary) seasonality, an appropriate model was employed. The prior model assumed that the rate of stroke cases varied arbitrarily in each month, with no

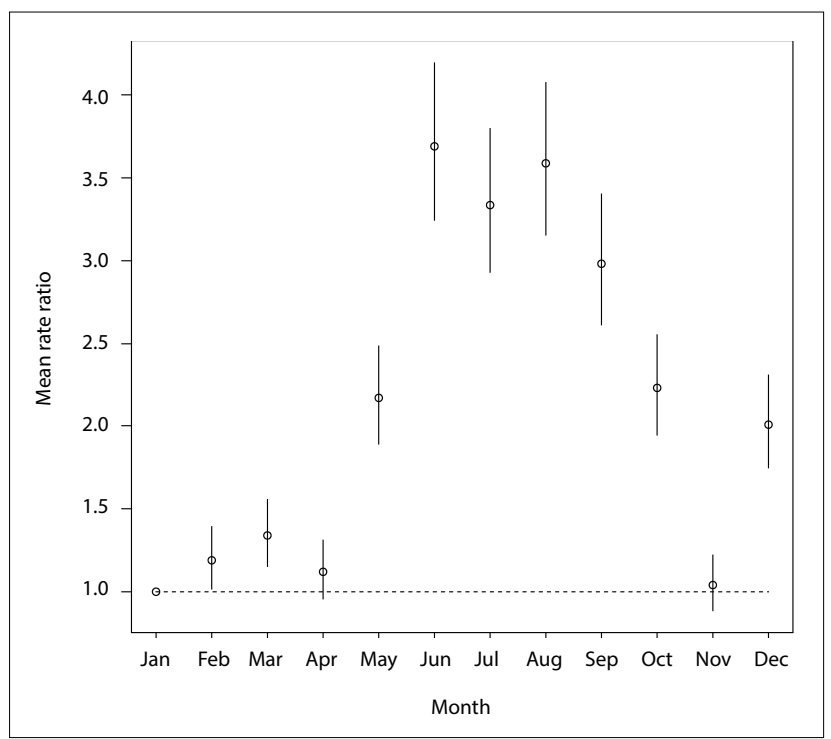

Fig. 3. Mean rate ratios and $95 \%$ confidence intervals of suspected stroke cases, using December as reference month.

Table 5. Monthly totals of suspected stroke cases over the 4 years of the study, according to risk factors ${ }^{\star}$

\begin{tabular}{|c|c|c|c|c|c|c|c|c|c|c|c|c|c|}
\hline Risk factor & Jan & Feb & Mar & Apr & May & Jun & Jul & Aug & Sep & Oct & Nov & Dec & Total \\
\hline Hypertension & 150 & 145 & 216 & 191 & 210 & 1110 & 1013 & 976 & 354 & 482 & 315 & 239 & 5401 \\
\hline Raised cholesterol & 392 & 422 & 381 & 290 & 491 & 389 & 562 & 1034 & 1064 & 546 & 367 & 310 & 6248 \\
\hline Heart problems & 213 & 206 & 298 & 263 & 399 & 1359 & 1445 & 1560 & 693 & 581 & 361 & 184 & 7562 \\
\hline Diabetes & 298 & 281 & 330 & 352 & 572 & 1741 & 1654 & 1635 & 693 & 675 & 399 & 228 & 8777 \\
\hline Total & 1053 & 1056 & 1225 & 1096 & 1672 & 4599 & 4674 & 5205 & 2804 & 2284 & 1442 & 961 & 19082 \\
\hline
\end{tabular}


smooth correlation between neighbouring months. The non-stationary seasonality model, which assumes the opposite, gave insignificant results. The insignificance of the non-stationary seasonality model confirms the stationary seasonality model as appropriate with a seasonal change variation of $\sim 5$ monthly stroke cases. However, the trend is gradually increasing. The results are displayed graphically in Fig. 4.

\section{Table 6. Cosinor test for seasonality of monthly confirmed stroke cases}

\begin{tabular}{ll}
\hline Observations, $N$ & 48 \\
Amplitude (absolute scale) & 120.05 (size of seasonal change) \\
Peak month & 7.50 (approx. mid-winter) \\
Low point: month & 1.50 (approx. mid-summer)
\end{tabular}

Table 7. Mean monthly variation in stroke cases

\begin{tabular}{llll}
\hline Month & Mean rate ratio & $\mathbf{9 5 \%} \mathbf{C I}$ & $\boldsymbol{p}$-value \\
\hline Feb & 1.19 & $1.02-1.39$ & 0.03 \\
Mar & 1.34 & $1.15-1.56$ & $<0.0001$ \\
Apr & 1.12 & $0.96-1.31$ & 0.16 \\
May & 2.17 & $1.89-2.49$ & $<0.0001$ \\
Jun & 3.69 & $3.24-4.20$ & $<0.0001$ \\
Jul & 3.33 & $2.93-3.80$ & $<0.0001$ \\
Aug & 3.59 & $3.16-4.08$ & $<0.0001$ \\
Sep & 2.98 & $2.61-3.40$ & $<0.0001$ \\
Oct & 2.23 & $1.94-2.55$ & $<0.0001$ \\
Nov & 1.04 & $0.89-1.22$ & 0.63 \\
Dec & 2.01 & $1.75-2.31$ & $<0.0001$ \\
CI $=$ confidence interval. & & &
\end{tabular}

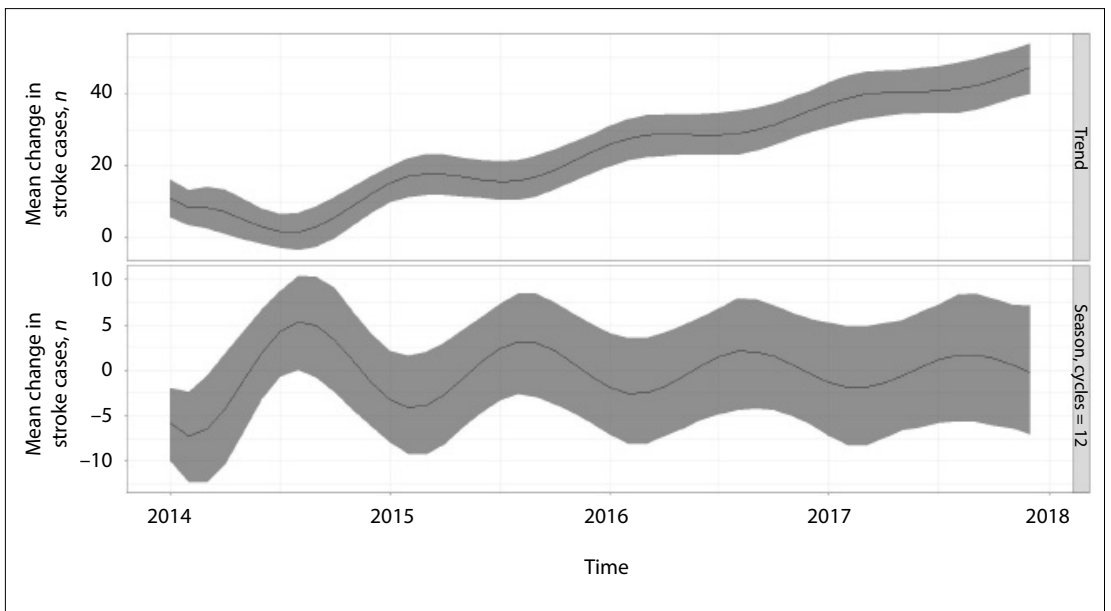

Fig. 4. Estimated trend and stationary seasonal pattern in stroke cases.

Having established the seasonal and trend patterns, we will focus on the predictors of stroke cases in the next section.

\section{Modelling the conditional mean of stroke cases}

The Poisson and negative binomial GLM analysis were considered in modelling stroke cases with risk factors as predictors, since the data are counts of stroke cases. The latter model caters for possible over-dispersion often inherent in data. In the GLM we include the linear trend and seasonality parameters to cater for both phenomena already confirmed above. Also, a first-order autoregressive parameter was included to cater for autocorrelation.

Table 8 shows the estimated Poisson GLM regression coefficients for the fitted model. This output presents first-order autocorrelation $\left(\beta_{1}\right)$, seasonality $\left(\beta_{13}\right)$, linear trend, hypertension, cholesterol, heart problems and diabetes as significant predictors of stroke (as all the confidence intervals do not include 0), except for cholesterol, which is marginally insignificant. The coefficient $\beta_{1}$ corresponds to regression on the previous observation, i.e. a first-order autocorrelation, and the coefficient $\beta_{13}$ captures seasonality, the unobserved conditional mean 13 time units (which is $\sim 1$ year) back in time. The predictor coefficients and their respective 95\% CIs are: $\beta_{1}$ (95\% CI 0.0725 - 0.1453), $\beta_{13}(95 \% \mathrm{CI}-0.1440--0.0666)$, linear trend (95\% CI $0.0804-0.1415$ ), hypertension (95\% CI $-0.0008--0.0001)$, cholesterol $\left(95 \% \mathrm{CI}-5.21 \times 10^{-6}-0.0007\right)$, heart problems (95\% CI $0.0019-0.0027)$ and diabetes (95\% CI $0.0039-0.0048$ ). Note that all statistically significant predictors have CIs on the positive side of the real number line, resulting in increases in stroke cases, except for hypertension.

The estimated over-dispersion coefficient $\sigma^{2}$ of 0.0994 from the negative binomialbased GLM is small (close to zero), indicating

Table 8. Poisson generalised linear model coefficients for stroke predictors

\begin{tabular}{llll}
\hline Coefficients & Estimate & SE & 95\% CI \\
\hline Intercept & 3.6623 & 0.0952 & $3.4800-3.8489$ \\
$\beta_{1}$ & 0.1089 & 0.0186 & $0.0725-0.1453$ \\
$\beta_{13}$ & -0.1053 & 0.0198 & $-0.1440--0.0666$ \\
Linear trend & 0.1109 & 0.0156 & $0.0804-0.1415$ \\
Hypertension & -0.0005 & 0.0002 & $-0.0008--0.0001$ \\
Cholesterol & 0.0004 & 0.0002 & $-5.21 \times 10^{-6}-0.0007$ \\
Heart problems & 0.0023 & 0.0002 & $0.0019-0.0027$ \\
Diabetes & 0.0044 & 0.0002 & $0.0039-0.0048$ \\
SE $=$ standard error; $\mathrm{CI}=$ confidence interval; $\beta_{1}=$ autocorrelation; $\beta_{13}=$ seasonality. & &
\end{tabular}


Table 9. Bivariate logistic and Poisson regression analysis of each predictor and stroke ( $N=14645$ cases of suspected stroke)

\begin{tabular}{|c|c|c|c|c|c|c|c|c|}
\hline \multirow[b]{2}{*}{ Variables } & \multirow[b]{2}{*}{ Total } & \multirow[b]{2}{*}{$n(\%)$} & \multicolumn{3}{|c|}{ Logistic } & \multicolumn{3}{|c|}{ Poisson } \\
\hline & & & OR & 95\% CI for OR & $p$-value & PR & 95\% CI for PR & $p$-value \\
\hline \multicolumn{9}{|l|}{ Gender } \\
\hline Male & 6270 & $6270(48.5)$ & Ref. & & & Ref. & & \\
\hline Female & 8375 & 8375 (57.2) & 18.23 & $16.75-19.85$ & $<0.0001$ & 3.79 & $3.60-4.00$ & $<0.0001$ \\
\hline \multicolumn{9}{|c|}{ Hypertension } \\
\hline Yes & 5401 & 5401 (36.9) & Ref. & & & Ref. & & \\
\hline No & 9244 & $9244(62.1)$ & 3.29 & $3.06-3.54$ & $<0.0001$ & 1.94 & $1.84-2.05$ & $<0.0001$ \\
\hline \multicolumn{9}{|c|}{ Heart problems } \\
\hline Yes & 7562 & $7562(51.6)$ & 8.86 & $8.23-9.55$ & $<0.0001$ & 3.03 & $2.873-3.19$ & $<0.0001$ \\
\hline No & 7083 & $7083(48.4)$ & Ref. & & & Ref. & & \\
\hline \multicolumn{9}{|c|}{ Raised cholesterol } \\
\hline Yes & 6248 & $6248(42.7)$ & Ref. & & & Ref. & & \\
\hline No & 8397 & 8397 (57.3) & 2.25 & $2.10-2.40$ & $<0.0001$ & 1.54 & $1.461-1.61$ & $<0.0001$ \\
\hline \multicolumn{9}{|l|}{ Diabetes } \\
\hline Yes & 8777 & 8777 (59.9) & 14.53 & $13.36-15.79$ & $<0.0001$ & 3.28 & $3.117-3.45$ & $<0.0001$ \\
\hline No & 5868 & $5868(40.1)$ & Ref. & & & Ref. & & \\
\hline \multicolumn{9}{|l|}{ Age (years) } \\
\hline $18-39$ & 1931 & $1931(13.2)$ & Ref. & & & Ref. & & \\
\hline $40-58$ & 2613 & $2613(17.8)$ & 0.92 & $0.83-1.03$ & 0.13 & 0.96 & $0.88-1.04$ & 0.264 \\
\hline $59-77$ & 5388 & $5388(36.8)$ & 1.37 & $1.24-1.50$ & $<0.0001$ & 1.172 & $1.10-1.25$ & $<0.0001$ \\
\hline $78-98$ & 4713 & 4713 (32.2) & 1.25 & $1.16-1.35$ & $<0.0001$ & 1.122 & $1.06-1.19$ & $<0.0001$ \\
\hline \multicolumn{9}{|l|}{ Race } \\
\hline Other & 4463 & $455(3.1)$ & Ref. & & & Ref. & & \\
\hline White & 10182 & $10182(69.5)$ & 2.00 & $1.86-2.15$ & $<0.0001$ & 1.47 & $1.39-1.55$ & $<0.0001$ \\
\hline
\end{tabular}

Table 10. Multivariate logistic regression model: gender, age, hypertension, cholesterol, heart problems, diabetes and race as predictors of stroke

\begin{tabular}{llll}
\hline Variables & OR & $\mathbf{9 5 \%}$ CI & p-value \\
\hline Gender & 0.83 & $0.75-0.93$ & 0.001 \\
Age & 1.08 & $1.03-1.14$ & 0.002 \\
Hypertension & 0.23 & $0.21-0.28$ & $<0.0001$ \\
Cholesterol & 3.54 & $2.96-4.22$ & $<0.0001$ \\
Heart problems & 0.07 & $0.06-0.08$ & $<0.0001$ \\
Diabetes & 0.05 & $0.04-0.06$ & $<0.0001$ \\
Race & 0.81 & $0.78-0.84$ & $<0.0001$ \\
OR = odds ratio; $\mathrm{CI}=$ confidence interval. & &
\end{tabular}

that there is no over-dispersion. There is therefore no need to consider this model.

\section{Bivariate models}

Table 9 shows that the likelihood (logistic) of stroke being significantly increased or reduced is associated with specific predictors.

For modifiable risk factors, the likelihood of stroke was significantly increased among all sampled patients with heart problems (odds ratio (OR) 8.86; 95\% CI 8.23 9.55; $p<0.0001)$ and diabetes (OR 14.53; 95\% CI 13.36 - 15.79; $p<0.0001$ ), with the reference group as no-stroke cases, while for non-modifiable risk factors, stroke was significantly increased among females
(OR 18.23; 95\% CI 16.75 - 19.85; $p<0.0001$ ), those aged $59-77$ years (OR 1.37; 95\% CI $1.24-1.50 ; p<0.0001)$ and $78-98$ years (OR 1.25; 95\% CI $1.16-1.35 ; p<0.0001$ ), and white patients (OR 2.00; 95\% CI 1.86 2.15; $p<0.0001$ ), with the reference groups as age group 18 - 39 years and other races, respectively. The likelihood of stroke was not significantly increased or reduced among stroke patients aged 40 - 58 years (OR 0.92; 95\% CI $0.83-1.03 ; p=0.13)$ compared with the reference group (18 - 39 years).

This study indicates that with regard to modifiable risk factors, individuals with heart problems were 8.86 times more likely to suffer from stroke than those without heart problems, while those with diabetes were 14.53 times more likely to suffer from stroke than those without diabetes. With regard to demographic factors, females were 18.23 times likely to suffer from stroke than males, and patients in the age groups $59-77$ years and $78-98$ years were 1.37 times and 1.25 times likely, respectively, to suffer from stroke than those in the age group 18 - 39 years.

In terms of the statistical significance of the prevalence ratios, the proportions of stroke cases as measured by Poisson regression analysis are in agreement with logistic regression results for the same respective risk factors.

\section{Multiple logistic regression analysis}

Multiple logistic regression analysis was carried out to investigate the relationship between stroke cases and the set of seven predictors simultaneously. The predictors were gender, age, hypertension, cholesterol, heart problems, diabetes and race. Table 10 shows that the predictors associated with comparatively reduced stroke cases were gender (OR 0.83; 95\% CI 0.75 - 0.93; $p=0.001$ ), hypertension (OR $0.23 ; 95 \%$ CI $0.21-0.28 ; p<0.0001)$, heart problems (OR 0.07; 95\% CI $0.06-0.08 ; p<0.0001$ ), 
diabetes (OR 0.5; 95\% CI $0.04-0.06 ; p<0.0001$ ) and race (OR 0.81 ; $95 \%$ CI $0.78-0.84 ; p<0.0001)$. On the other hand, those that were associated with a comparative increase in stroke cases were age (OR 1.08; 95\% CI $1.03-1.14 ; p=0.002$ ) and raised cholesterol (OR 3.5; $95 \%$ CI $2.96-4.22 ; p<0.0001)$.

\section{Discussion}

The present study investigated the trend and monthly seasonal variations in stroke cases in SA, as well as their potential predictors. A total of 14645 suspected stroke cases were retrieved from the hospital databases, of which 7538 (51.5\%) were confirmed. The study showed a significant trend, first-order autocorrelation and seasonality in stroke cases. The rate of stroke cases was highest in winter, followed by spring, summer and lastly autumn. The winter month with the peak number of cases of stroke was June, and the peak spring month was September. These findings are consistent with a study by Raj et al. ${ }^{[15]}$ Few studies have demonstrated no significant seasonal variations in stroke cases or identified summer as the season with most cases of stroke. ${ }^{[1,4,17]}$ Similar results to ours, showing high rates of stroke in winter and spring, were obtained in Poland and Hungary. ${ }^{[18]}$ The above finding also corresponds with our results and those of Moinuddin et al. ${ }^{[19]}$

Stroke, like any other non-communicable disease, has a complex, multifactorial aetiology. ${ }^{[20]}$ The roles of non-modifiable factors such as age, sex and race have been pointed out in several studies, whereas smoking, drinking alcohol, physical inactivity and diet can be modified to reduce the risk of stroke significantly. The predisposition to stroke in winter appears to be multifactorial, with increased serum concentrations of plasma fibrinogen and total cholesterol reported by Gordon et al. ${ }^{[21]}$ A study in Belgium found an increase in cases of stroke in the late autumn and winter owing to the effect of low temperatures on blood pressure. ${ }^{[22]}$ Cholesterol levels have been found to be higher in winter than in summer. ${ }^{[23]}$ In contrast, Shigematsu et al. ${ }^{[24]}$ found that elevated blood pressure was due to stress, and that type 2 diabetes mellitus and age were the most common risk factors for stroke. Similarly, our results showed that $42.7 \%$ of stroke patients had high cholesterol levels, $86.9 \%$ had diabetes and $75.7 \%$ had heart problems. While hypertension, raised cholesterol and diabetes have frequently been reported as lifestyle risk factors for stroke, age and gender have been identified as common non-modifiable risk factors. ${ }^{[23]}$ In a 5-year study, Wang et $a l .{ }^{[11]}$ consistently found half of their stroke patients to be in the age group 60 - 69 years, with fewer cases at younger ages (20 - 44 years). Similarly, a study in Korea found that high proportions of ischaemic stroke occurred in older age groups ${ }^{[13]}$ and that the prevalence of cases in the age group 18 - 39 years was insignificant. Studies in other countries have had similar findings. ${ }^{[4,11,15]}$

The biological reasons for the increased occurrence of stroke cases in winter have not yet been ascertained, but several mechanisms may be proposed. It is well known that blood pressure varies according to season, with higher levels in winter, possibly due to cold-induced peripheral blood vessel constriction, ${ }^{[22]}$ and increased blood pressure and raised cholesterol have been suggested as major reasons for the increase in stroke cases in winter. ${ }^{[16,20]}$

Since the real mechanisms underlying the variation in risk factors for stroke are unclear, further studies are needed to better understand this issue. Such studies may help in developing preventive campaigns. Conflicting evidence has been reported on the association between risk factors and stroke cases. Although some evidence exists for higher numbers of stroke cases in winter due to raised cholesterol, hypertension and diabetes, others have failed to detect any modifiable risk factors. The present study supports studies that showed an increase in stroke cases in winter and spring. ${ }^{[19,20]}$

\section{Study limitations}

A large proportion of the data used in this study were retrieved from the databases of the private hospitals, as the public hospitals were not capturing all the pertinent variables. However, most South Africans (70\%) use public hospitals. This shortcoming was addressed by a use of a stratified probability sampling technique to calculate the proportions accordingly. This study randomly selected $33 \%$ of the 203 private hospitals in all nine provinces of SA, while $67 \%$ of the 407 public hospitals were randomly selected across the provinces. The proportions were calculated based on the total number of public and private hospitals in SA.

\section{Conclusions}

In summary, gender, age, race, diabetes and heart problems were found to be significant risk factors for stroke in the present study. Diabetes and heart problems are modifiable risk factors, while gender, race and age are not. Modifiable risk factors can be managed by avoiding excessive weight gain, reducing salt and sugar intake and exercising regularly.

The study findings call for programmes and policies to curb unhealthy lifestyles and promote physical activity and healthy nutrition, starting in childhood. We recommend that wellness centres be set up at schools, tertiary institutions and workplaces and in communities, where people can be tested regularly for hypertension, raised cholesterol and diabetes. Health professionals should raise awareness of the dangers of these risk factors. In addition, the tax on cigarettes, alcohol and unhealthy foods could be increased in an effort to discourage their consumption.

We found that there was a distinct seasonal variation in cases of stroke, with a higher prevalence during winter and spring. Monthly variation in stroke was most prominent in women, individuals with raised cholesterol, and the younger age group. The risk factors of raised cholesterol, hypertension and diabetes were associated with more cases in June, July and August than in the summer months. However, the biological mechanisms underlying these increases remain unclear. Further studies based on our findings are needed to enhance understanding of stroke and its association with gender, hypertension, cholesterol and age. A holistic approach to arresting the increasing trend of stroke should take into account both seasonality and the associated risk factors.

Declaration. None.

Acknowledgements. The authors thank the National Research Foundation (NRF) for funding this work, and the SAMJ editor and referees for careful reading and comments that greatly improved the article.

Author contributions. ER conceptualised the study and did the R-code programming. LM did the sampling design and data collection, while data interpretation, drafting and revisions of the article were carried out by both ER and LM.

Funding. This work was supported by the NRF of SA.

Conflicts of interest. None.

1. Bahonar A, Khosravi A, Khorvash F, Maracy M, Saadatnia M. Seasonal and monthly variation in stroke and its subtypes - 10-year hospital-based study. Mater Sociomed 2017;29(2):119-123. https:// stroke and its subtypes - 10-year
doi:10.5455/msm.2017.29.119-123

2. Sanya EO, Desalu OO, Adepoju F, Aderibigbe SA, Shittu A, Olaosebikan O. Prevalence of stroke in semi-urban communities in middle-belt region of Nigeria: A door to door survey. Pan Afr Med J 2015;20(33):1-8. https://doi.org/10.11604/pamj.2015.20.33.4594 
3. Owolabi MO, Akarolo-Anthony S, Akinyemi R, et al. The burden of stroke in Africa: A glance at the present and a glimpse into the future. Cardiovasc J Afr 2015;20(2):S27-S38. https://doi.org/10.5830/ CVJA-2015-038

4. Zamani P, Mousavi SM, Rezai H, Madjdinasab N. Circadian variation in the incidence of strokeinduced aphasia types in Ahvaz, South-West Iran. Jentashapir J Health Res 2015;6(6):27-31. https:// induced aphasia types in Ahra
doi.org/10.17795/jijr-28710

5. Mudzi W, Stewart A, Musenge E. Case fatality of patients with stroke over a 12-month period post stroke. S Afr Med J 2012;102(9):1-8. https://doi.org/10.7196/SAMJ.5742

6. Maredza M, Chola L. Economic burden of stroke in rural South Africa setting. eNeurologicalSci 2016;3:26-32. https://doi.org/10.1016/j.ensci.2016.01.001

7. Maredza M, Bertram MY, Tollman SM. Disease burden of stroke in rural South Africa: An estimate of 7. Maredza M, Bertram MY, Tollman SM. Disease burden of stroke in rural South Africa: An estimate of
incidence, mortality and disability adjusted life years. BMC Neurol 2015;15(54):1-12. https://10.1186 incidence, mortality

8. Ovbiagele B, Nguyen-Huynh MN. Stroke epidemiology: Advancing our understanding of disease mechanism and therapy. Neurotherapeutics 2011;8(3):319-329. https://doi.org/10.1007/s13311-0110053-1

9. Wandai M, Aagaard-Hansen J, Day C, Sartorius B, Hofman K. Available data sources for monitoring non-communicable diseases and their risk factors in South Africa. S Afr Med J 2017;107(4):331-337. https://doi.org/10.7196/SAMJ.2017.v107i4.11438

10. Barnett AG, Dobson AJ. Analysing Seasonal Health Data. Berlin: Springer-Verlag, 2010. https://doi. org/10.1007/978-3-642-10748-1-2

11. Wang Y, Levi CR, Attia JR, D'Este CA, Spratt N, Fisher J. Seasonal variation in stroke in the Hunter Region, Australia: A 5-year hospital-based study 1995 - 2000. J Am Heart Assoc 2003;34(5):1144-1150. https://doi.org/10.1161/01.STR.0000067703.71251.B6

12. Anderson N, Feigin V, Bennet D, et al. Diurnal, weekly, and seasonal variations in stroke occurrence in a population-based study in Auckland, New Zealand. N Z Med J 2012;117(1202):1-8.

13. $\mathrm{Han} \mathrm{MH}$, Yi HJ, Kim YS. Effect of seasonal and monthly pollution factors on stroke incidence in Seoul, Korea. Stroke 2015;41(4):927-935. https://doi.org/10.1161/STROKEAHA.114.007950

14. Christensen AL, Rasmussen LH, Baker MG, Lip GYH. Seasonality, incidence and prognosis in atrial fibrillation and stroke in Denmark and New Zealand. BMJ Open 2012;2(10):1-8. https///dol. org/10.1136/bmjopen-2012-001210
15. Raj K, Bhatia R, Prasad K, Srivastava MVP, Vishnubhatla S, Singh MB. Seasonal differences and circadian variation in stroke occurrence and stroke subtypes. J Stroke Cerebrovasc Dis 2015;24(1):1016. https://doi.org/10.1016/j.jstrokecerebrovasdis.2014.07.051

16. Statistics South Africa. Mid-year population estimates, 2015. Statistical release P0302. https://www. statssa.gov.za/publications/P0302/P03022015.pdf (accessed 1 May 2018).

17. Xu B, Liu H, Su N, et al. Associations between winter season and risk of death from cardiovascular diseases: A study in more than half a million inpatients in Beijing, China. BMC Cardiovasc Disord 2013;13:93. https://doi.org/10.1186/1471-2261-13-93

18. Klimaszewska K, Kulak K, Jankowiak B, Kowalczuk K, Kondzior D, Baranowsk A. Seasonal variation in ischaemic stroke frequency in Podlaskie Province by season. Adv Med Sci 2007;52(1):112-114

19. Moinuddin A, Shara N, Lewsey J, Inglis, S. Effect of seasonal variation on the frequency of incident stroke hospitalizations in Scotland. Saudi J Health Sci 2015;4(1):23-26. https://doi.org/10.4103/22780521.151405

20. Geber Y, Jacobsen SJ, Killian JM, Weston SA, Roger VL. Seasonality and daily weather conditions in relation to myocardial infarction and sudden cardiac death in Olmsted County, Minnesota, 1979 to 2002. J Am Coll Cardiol 2006;48(2):287-292. https://doi.org/10.1016/j.jacc.2006.02.065

21. Gordon DJ, Hyde J, Trost DC. Cyclical seasonal variations in plasma lipid and lipoprotein level: The Lipid Research Clinics Coronary Primary Prevention Trial placebo group. J Clin Epidemiol 1988;41(7):679-689. https://doi.org/10.1016/0895-4356(88)90120-5

22. Capon A, Demeurisse G, Zheng 1. Seasonal variations of cerebral hemorrhage in 236 consecutive cases in Brussels. Stroke 1992;23(1):24-27. https://doi.org/10.1161/01.str.23.1.24

23. Joseph TD, Sandra HK, David FB. Seasonal variation in serum cholesterol concentration. J Chronic Dis 1965;18:657-664. https://doi.org/10.1016/0021-9150(92)90171-c

24. Shigematsu K, Watanabe Y, Nakano H. Weekly variations of stroke occurrence: An observational cohort study based on the Kyoto stroke registry, Japan. BMJ Open 2015;5(3):e006294. https://doi. org/10.1136/bmjopen-2014-006294

Accepted 27 September 2019. 